\title{
Subacute thyroiditis presenting as acute psychosis: a case report and literature review
}

\author{
Kyung Ae Lee, Kyung Taek Park, Hea Min Yu, Heung Yong Jin, Hong Sun Baek, and Tae Sun Park
}

Division of Endocrinology and Metabolism, Department of Internal Medicine, Research Institute of Clinical Medicine of Chonbuk National University and Chonbuk National University Hospital, Jeonju, Korea

\author{
Received: August 4, 2011 \\ Revised : September 30, 2011 \\ Accepted: October 24, 2011

\section{Correspondence to} \\ Tae Sun Park, M.D. \\ Division of Endocrinology and \\ Metabolism, Department of \\ Internal Medicine, Chonbuk \\ National University Medical \\ School, 20 Geonji-ro, Deokjin-gu, \\ Jeonju 561-712, Korea \\ Tel: +82-63-250-1794 \\ Fax: +82-63-254-1609 \\ E-mail:pts@jbnu.ac.kr
}

We describe herein an unusual case of subacute thyroiditis presenting as acute psychosis. An 18-year-old male presented at the emergency department due to abnormal behavior, psychomotor agitation, sexual hyperactivity, and a paranoid mental state. Laboratory findings included an erythrocyte sedimentation rate of $36 \mathrm{~mm} / \mathrm{hr}$ (normal range, o to 9), free $\mathrm{T}_{4}$ of $100.0 \mathrm{pmol} / \mathrm{L}$ (normal range, 11.5 to 22.7), and thyroid stimulating hormone of $0.018 \mathrm{mU} / \mathrm{L}$ (normal range, 0.35 to 5.5). A technetium-99m pertechnetate scan revealed homogeneously reduced activity in the thyroid gland. These results were compatible with subacute thyroiditis, and symptomatic conservative management was initiated. The patient's behavioral abnormalities and painful neck swelling gradually resolved and his thyroid function steadily recovered. Although a primary psychotic disorder should be strongly considered in the differential diagnosis, patients with an abrupt and unusual onset of psychotic symptoms should be screened for thyroid abnormalities. Furthermore, transient thyroiditis should be considered a possible underlying etiology, along with primary hyperthyroidism.

Keywords: Psychotic disorders; Thyroiditis, subacute; Thyrotoxicosis

\section{INTRODUCTION}

Triiodothyronine $\left(\mathrm{T}_{3}\right)$ receptors are distributed throughout the brain, especially in the limbic system, and they seemingly support a variety of functions including emotion, behavior, and long term memory [1]. Evidence suggests that the modulation of the adrenergic receptor response to catecholamines by thyroid hormones in the central nervous system (CNS) may lead to psychotic behavior in thyrotoxic patients [2]. As a result, persons with thyroid dysfunction frequently experience a wide variety of neuropsychiatric sequelae. However, most medical texts do not mention psychosis as a presenting feature of thyrotoxicosis. Additionally, most cases of psychosis caused by thyrotoxicosis or hyperthyroidism have been described in patients with Graves' disease or toxic multinodular goiter. Herein, we report a case of an 18 -year-old male with subacute thyroiditis who presented with acute psychosis.

\section{CASE REPORT}

An 18-year-old male presented to the emergency department due to abnormal behavior, agitation, and increased activity. One week prior to this visit, the patient had reported experiencing dizziness, headache, and sore throat, for which he had taken a cold medicine which had included a nonsteroidal anti-inflammatory drug. Three days prior to the emergency department visit, the patient had reported experiencing anxiety, irritability, and restlessness, which subsequently worsened to additional features of psychomotor agitation and a paranoid mental state. The patient 
was healthy with the exception of these psychomotor problems; he reported doing well in school and maintained good interpersonal relationships. There was no history of recent or past alcohol or drug abuse and there was no family history of psychiatric disorders.

On arrival, the patient appeared agitated and had a poor attention span. He complained of a sore throat, febrile sensation, and dizziness. He was irritable and threatening, as well as talkative. His speech was irrational and seemingly random. The patient also exhibited persecutory delusions toward his mother. Physical examination revealed tachycardia with a heart rate over 100 beats/min, moist skin, and an elevated body temperature $\left(37.8^{\circ} \mathrm{C}\right)$. Chest and abdominal examinations revealed no abnormalities.

Laboratory findings included a leukocyte count of $6.02 \times 10^{9} / \mathrm{L}$, an erythrocyte sedimentation rate (ESR) of $36 \mathrm{~mm} / \mathrm{hr}$ (normal range, o to 9), aspartate aminotransferase of $38 \mathrm{IU} / \mathrm{L}$, alanine aminotransferase of 62 $\mathrm{IU} / \mathrm{L}$, and high sensitive C-reactive protein of $0.21 \mathrm{mg} /$ $\mathrm{L}$ (normal range, o to 5). Brain computed tomography and subsequent lumbar puncture revealed no abnormal findings. Thyroid function tests revealed a thyrotoxic state with free $\mathrm{T}_{4}$ of $100.0 \mathrm{pmol} / \mathrm{L}$ (normal range, 11.5 to 22.7 ) and thyroid stimulating hormone (TSH) of $0.018 \mathrm{mU} / \mathrm{L}$ (normal range, 0.27 to 4.2 ), antithyroid peroxidase antibody and anti-TSH receptor antibody testing were negative. The patient's thyroid gland was hard and mildly enlarged, and he reported tenderness on palpation. Subacute thyroiditis was suspected, but the patient's uncooperative state prohibited further diagnostics. We subsequently consulted the psychiatry department. According to the clinical symptoms and laboratory results, a psychiatrist diagnosed the patient with a mood disorder due to a general medical condition (Diagnostic and Statistical Manual of Mental Disorders 4th edition, 293.83), and the accompanying symptoms were presumed to be the result of a thyrotoxic state caused by subacute thyroiditis.

The patient was admitted to a closed ward in the psychiatric department for careful observation. Symptomatic treatment and heart rate control with a beta blocker (propranolol $20 \mathrm{mg}$ twice daily), was continued, and antipsychotic drugs, including haloperidol $5 \mathrm{mg}$ daily and lorazepam $4 \mathrm{mg}$ daily, were also used. Over the first 3 days of admission, the patient's increased activity, especially sexual hyperactivity, and offensive attitude did not subside, but after 2 weeks, marked improvement was observed in all symptoms. Subsequent blood tests revealed improved thyroid function with a free $\mathrm{T}_{4}$ of $41.4 \mathrm{pmol} / \mathrm{L}$, and TSH of 0.01 $\mathrm{mU} / \mathrm{L}$. The patient's overall condition was markedly better and he was more cooperative. Therefore, thyroid ultrasonography and a technetium-99m ( $\left.{ }^{99 \mathrm{~m}} \mathrm{Tc}\right)$ scan were performed. The sonographic findings included multifocal heterogeneous echogenicity with normal gland size and decreased vascularity (Fig. 1). The ${ }^{99 \mathrm{~m}} \mathrm{Tc}$ pertechnetate scan revealed homogenously reduced Tc uptake in the thyroid gland (20 minutes Tc-pertechnetate uptake, 1.1\%) (Fig. 2).

Approximately 20 days after hospitalization, the patient's free $\mathrm{T}_{4}$ level had decreased to nearly normal and
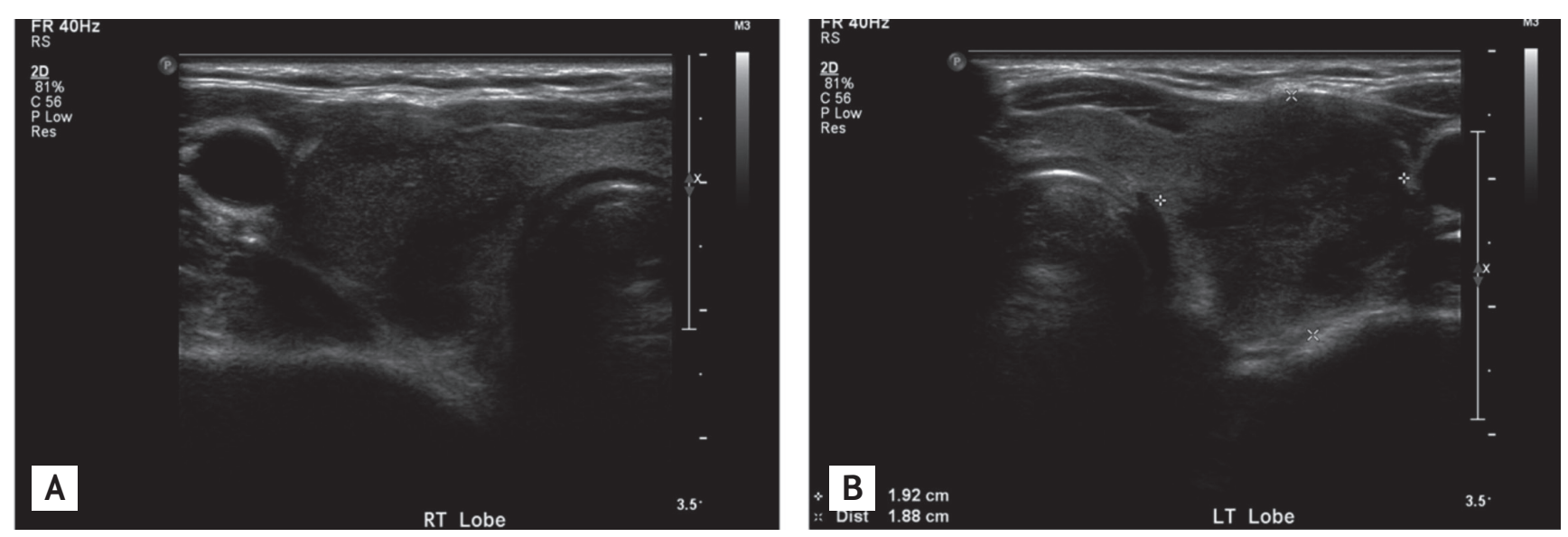

Figure 1. (A, B) Thyroid ultrasonography. Ultrasonography revealed multifocal heterogeneous lesions with normal gland size and decreased vascularity. 
he was gradually weaned off the antipsychotics before being subsequently discharged. On follow-up, the patient remained asymptomatic and has since returned to school without recurrence of psychiatric symptoms (Fig. 3).

\section{DISCUSSION}

Subacute thyroiditis, a self-limiting inflammatory thyroid disorder usually caused by a viral infection, most often presents with thyroid pain and systemic symptoms. The clinical features include thyrotoxicosis, suppressed levels of TSH, decreased thyroid uptake of radioactive iodine, and an elevated ESR. Common symptoms of thyrotoxicosis include heat intolerance, excessive sweating, weight loss, palpitations, and gastrointestinal upset. Various psychiatric symptoms such as anxiety, emotional lability, insomnia, agitation, mania, and depression are also concurrent with hypermetabolic symptoms. Nevertheless, psychosis and acute confusion are rare as an initial presentation.

There is little doubt that thyroid hormone plays a major role in the regulation of mood, cognition, and behavior. Indeed, persons with thyroid dysfunction frequently experience a wide variety of neuropsychiatric sequelae. However, the pathogenesis of psychosis in thyrotoxicosis is remains to be explained. The brain appears to possess a unique sensitivity to thyroid hormone and to utilize thyroid hormone differently from other organ systems [3]. High concentrations of $\mathrm{T}_{3}$ receptors are found in the limbic system (especially the amygdala and hippocampus), and seemingly support a variety of functions including emotion, behavior, and long-term memory. Another possible mechanism involves excess thyroid hormone affecting $r$ zurotransmitters such as serotonin, dopamine, or second messengers [4]. Evidence suggests that modulation of the beta-adrenergic receptor response to catecholamines by thyroid hormones may also contribute to these effects. Thyroid hormones increase the ability of these receptors to receive stimulation. Such interaction between catecholamines and thyroid hormones in the CNS is strengthened by their common origin from the amino acid tyrosine and their synergism in many metabolic processes [2].

In 1835, in Graves' classic description of the disorder that now bears his name, he focused on nervous dysfunction, suggesting a relationship between the thyroid gland with the syndrome of globus hystericus
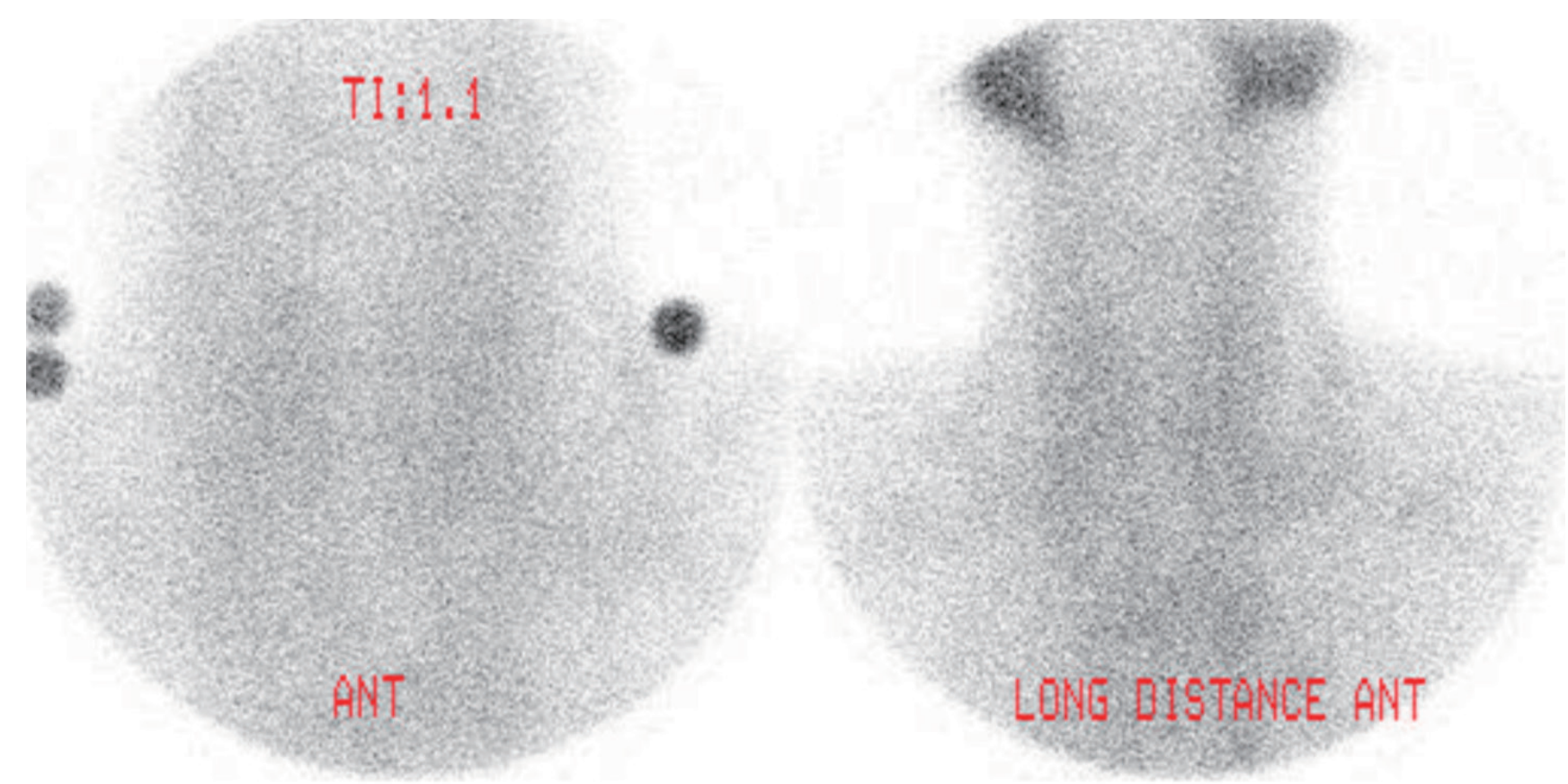

Figure 2. Technetium- $99 \mathrm{~m}\left({ }^{99 \mathrm{~m}} \mathrm{Tc}\right)$ pertechnetate thyroid scan. The ${ }^{99 \mathrm{~m}} \mathrm{Tc}$ pertechnetate scan demonstrated homogenously reduced Tc uptake in the thyroid gland (20 minutes Tc-pertechnetate uptake, 1.1). 
[5]. In 1840, Basedow provided the first description of associated psychosis, but it was not until 1886 that a thyrotoxic syndrome of endocrine origin was clearly distinguished from a group of neuroses [6]. Brownlie et al. [7] reported a series of newly diagnosed thyrotoxic patients with concurrent acute psychosis which included 11 cases of Graves' disease, six cases of toxic multinodular goiter, and one case of toxic adenoma. They suggested that thyrotoxicosis is a precipitant of acute affective psychosis. Several additional cases of psychological illness associated with thyrotoxicosis have been reported since (Table 1). Furthermore, psychiatric disorders often accompany hypothyroidism. Psychosis associated with hypothyroidism, 'myxedema madness' is generally accepted as a real phenomenon. The occurrence of more severe psychiatric features depends upon the severity and duration of disease and the underlying predisposition of the individual to psychiatric instability.

Most cases of thyroid-related psychosis have been described in patients with Graves' disease or toxic multinodular goiter; however, thyrotoxicosis associated with transient thyroiditis or factitious thyrotoxicosis can also result in severe behavioral disturbances, including organic psychosis. Two cases of acute psychotic episodes in patients with thyrotoxicosis factitia and subacute thyroiditis have been reported $[8,9]$. In both cases, psychotic features occurred abruptly and resolved with resolution of the thyrotoxic state. Furthermore, the thyroidal inflammation characteristic of subacute thyroiditis, can cause schizophrenia, mania, psychotic depression, and paranoid behavior severe enough to require inpatient treatment.
While we considered that the coexistence of psychosis and subacute thyroiditis could have been a chance occurrence, we diagnosed our patient with psychosis due to subacute thyroiditis for three reasons. First, the appearance of psychiatric symptoms was chronologically related to the onset of thyrotoxicosis and the resolution of the psychosis was temporally related to the attainment of biochemical euthyroidism and amelioration of the tachycardia (Fig. 3). This suggested that the psychotic symptoms were directly related to the thyrotoxicosis. Second, the clinical symptoms of a sore throat and anterior neck tenderness, the laboratory results of an elevated ESR and an elevated free $\mathrm{T}_{4}$ level, and the typical findings on thyroid ultrasonography and scan were compatible with subacute thyroiditis. Third, there was no history of recent or past alcohol or drug abuse, and there was no family history of psychiatric disorders. Treatment with a $\beta$-adrenergic antagonist drug can be useful in controlling the anxiety associated with thyrotoxicosis in subacute thyroiditis. Furthermore, in acutely psychotic patients, dopamine blockade with a medicine such as haloperidol may be required to reduce excitement. As such, our patient was treated appropriately with propranolol, haloperidol, and lorazepam.

When dealing with such a case, it is important to make a precise diagnosis and exclude other possible diseases. Several medical conditions can cause mood disorders, including Cushing's syndrome, Addison's disease, thyroid abnormalities, diseases of the pancreas, rheumatoid arthritis, and infectious diseases such as mononucleosis and electrolyte disturbances. With the exception of thyrotoxicosis, our patient had

Table 1. Cases of acute psychosis associated with thyrotoxicosis

\begin{tabular}{|c|c|c|c|c|}
\hline Year published & Underlying disease & Number of patients & Mean age & $\operatorname{Sex}(n)$ \\
\hline 1935 & Graves' disease & 4 & 43 & $\mathrm{~F}(4)$ \\
\hline 1968 & Graves' disease & 1 & 28 & $\mathrm{M}$ \\
\hline \multirow[t]{2}{*}{2000} & Graves' disease & 11 & 54 & $\mathrm{M}(1), \mathrm{F}(10)$ \\
\hline & TMNG or toxic adenoma & 7 & 54 & $\mathrm{M}(1), \mathrm{F}(6)$ \\
\hline 2005 & Graves' disease & 1 & 25 & $\mathrm{M}$ \\
\hline 2009 & Graves' disease & 1 & 43 & $\mathrm{~F}$ \\
\hline 2009 & Graves' disease & 1 & 40 & $\mathrm{~F}$ \\
\hline
\end{tabular}

F, female; M, male; TNMG, toxic multinodular goiter. 


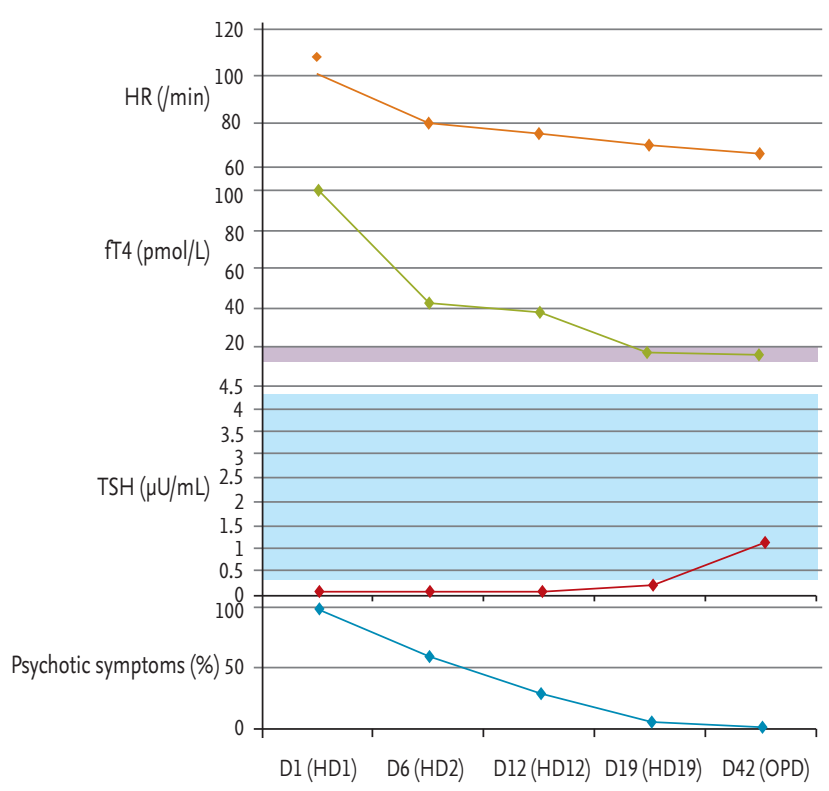

Figure 3. Clinical course of the patient during and after hospitalization. The appearance of psychiatric symptoms was chronologically related to the onset of thyrotoxicosis and the resolution of the psychosis was temporally related to the attainment of biochemical euthyroidism and amelioration of the tachycardia. $\mathrm{HR}$, heart rate; $\mathrm{fT}_{4}$, free $\mathrm{T}_{4}$; TSH, thyroid stimulating hormone; HD, hospitalization day; OPD, outpatient department.

no abnormal symptoms or laboratory results. It is also important to distinguish these cases from the hyperthyroxinemia that may accompany acute psychiatric disorders, including schizophrenia and mania. Free thyroxine elevation is modest and transient and is usually seen within 1 week. This entity has not been established clearly, but may be considered a form of nonthyroidal illness [10].

In conclusion, although a primary psychotic disorder should be considered in the differential diagnosis of patients who present with an abrupt onset of unusual psychotic symptoms, in the absence of a history of substance abuse, such patients should be screened for thyroid abnormalities or other underlying endocrine abnormalities. In particular, the presence of physical manifestations such as heat intolerance, excessive sweating, weight loss, and palpitations should raise suspicion of hyperthyroidism. A high index of suspicion is often required to detect the association. Furthermore, in addition to primary hyperthyroidism, transient thyroiditis should also be considered a possible etiology.

\section{Conflict of interest}

No potential conflict of interest relevant to this article is reported.

\section{REFERENCES}

1. Bunevicius R, Prange AJ Jr. Psychiatric manifestations of Graves' hyperthyroidism: pathophysiology and treatment options. CNS Drugs 2006;20:897-909.

2. Whybrow PC, Prange AJ Jr. A hypothesis of thyroidcatecholamine-receptor interaction: its relevance to affective illness. Arch Gen Psychiatry 1981;38:106-113.

3. Dratman MB. Cerebral versus peripheral regulation and utilization of thyroid hormones. In: Joffe RT, Levitt AJ, eds. The Thyroid Axis and Psychiatric Illness. Washington, DC: American Psychiatric Press, 1993:3-94.

4. Stern RA, Prange AJ. Neuropsychiatric aspects of endocrine disorders. In: Kaplan HI, Sadock BJ, eds. Comprehensive Textbook of Psychiatry. 6th ed. Baltimore: Wiliams \& Wilkins, 1995:241-251.

5. Graves RJ. Newly observed affection of the thyroid gland in females. Lond Med Surg J 1835;7:516-517.

6. Philippopoulos GS. Thyrotoxicosis and its psychosomatic approach. J Nerv Ment Dis 1959;128:415-424.

7. Brownlie BE, Rae AM, Walshe JW, Wells JE. Psychoses associated with thyrotoxicosis: 'thyrotoxic psychosis.' A report of 18 cases, with statistical analysis of incidence. Eur J Endocrinol 2000;142:438-444.

8. da Silva JA, Almeida JT, Correa BB, Narigao M, Xavier M. Acute psychotic episode in a patient with thyrotoxicosis factitia. BMJ Case Rep 2009;2009:bcro8.2008.0676.

9. Rizvi AA. "Thyrotoxic psychosis" associated with subacute thyroiditis. South Med J 2007;100:837-840.

10. Spratt DI, Pont A, Miller MB, McDougall IR, Bayer MF, McLaughlin WT. Hyperthyroxinemia in patients with acute psychiatric disorders. Am J Med 1982;73:41-48. 